\title{
LES ÉCRIVAINES DANS LES LITTÉRATURES MINEURES DES ÉTATS-UNIS: DU MELTING POT À LA GHETTOÏSATION
}

\author{
Carolina Ferrer ${ }^{1}$ \\ Roxane Maiorana ${ }^{2}$
}

\begin{abstract}
Résumé: Terre d'immigration, les États-Unis sont formés de nos jours de diverses communautés culturelles. Dans le champ littéraire étatsunien, ces différentes communautés se traduisent par une multiplicité de littératures mineures, telles que définies par Deleuze et Guattari (1975). Cette recherche a pour objectif d'analyser les relations qu'entretiennent celles-ci entre elles et avec le champ littéraire national. En particulier, nous étudions la place que les écrivaines occupent au sein de ces littératures mineures. Notre approche méthodologique se base principalement sur la criticométrie (Ferrer, 2011). À travers l'exploitation et la compilation de milliers de références en provenance de la base de données Modern Language Association International Bibliography, nous élaborons plusieurs indicateurs métacritiques. Dans un premier temps, nous analysons la place des femmes dans la littérature des États-Unis. Nous répertorions, dans un deuxième temps, les littératures mineures qui composent le champ littéraire de cette littérature nationale. Enfin, nous montrons les intrications qui façonnent les liens entre les écrivaines étatsuniennes et leur appartenance aux littératures mineures.
\end{abstract}

Mots-clés: Littérature mineure, littérature des États-Unis, criticométrie, écrivaines, ghettoïsation.

\section{WOMEN WRITERS IN MINOR LITERATURES OF THE UNITED STATES: FROM MELTING POT TO GHETTOIZATION}

\begin{abstract}
Land of immigration, the United States of America is composed of several cultural communities. In the American literary field, these communities result in multiple minor literatures as defined by Deleuze and Guattari (1975). The goal of this research is to analyze the relationships between these minor literatures and the national literary field. Particularly, we study the participation of women writers within these minor literatures.
\end{abstract}

\footnotetext{
1Professeure, Département d'études littéraires, Université du Québec à Montréal, ferrer.carolina@uqam.ca. Je tiens à remercier le Conseil de recherches en sciences humaines du Canada, qui finance le projet CRSH 435-2018-1115, intitulé « Les études littéraires et les nouveaux observables de l'ère numérique : le système de la littérature mondiale de l'après-guerre à nos jours », dans lequel s'inscrit cette étude. Endereço eletrônico: ferrer.carolina@uqam.ca

2 Doctorante, Programme de doctorat en études littéraires, Université du Québec à Montréal, maiorana.roxane@courrier.uqam.ca.
} 
Methodologically, we base our study on criticometrics (Ferrer, 2011). Through the exploitation and the compilation of thousands of references obtained from the Modern Language Association International Bibliography database, we elaborate several indicators. Firstly, we examine women's place in American literature. Secondly, we identify the minor literatures that constitute this national literature. Finally, we highlight the relationships between American women writers and their belonging to minor literatures.

Keywords: Minor Literature, American Literature, Criticometrics, Women Writers, Ghettoization.

On the boats and on the planes

They're coming to America

Never looking back again,

They're coming to America

Everywhere around the world

They're coming to America

Ev'ry time that flag's unfurled

They're coming to America

Got a dream to take them there They're coming to America Got a dream they've come to share

They're coming to America

«America»

Neil Diamond

\section{Terre d'immigrants, terre de littératures mineures}

Melting pot par excellence, les États-Unis se démarquent comme une société constituée par une multiplicité de communautés culturelles qui se sont formées au rythme des vagues d'immigration. Selon Yasmeen Abu-Laban et Victoria Lamont, la construction de la nation étatsunienne repose sur le mélange de ces dernières: "Alluding to the history of the United States as a settler-colony, the melting pot metaphor [...] refers to processes of immigration, relations of ethnic diversity, and notions of national identity and purpose. " (1997, p. 23) A priori, nous pourrions croire que chacune de ces différentes composantes possède une place bien établie dans l'ensemble du pays. Cependant, il semble qu'elles se structurent plutôt en ghettos. Alors que 
LES ÉCRIVAINES DANS LES LITTÉRATURES MINEURES DES ÉTATS-UNIS: DU MELTING POT À LA GHETTOÏSATION

ce dernier terme est intimement lié au traitement réservé à la diaspora juive en Europe (WACQUANT, 2005), aux États-Unis, historiquement, les ghettos ont été formés pour séparer physiquement la population étatsunienne noire de la blanche, matérialisant ainsi «la domination ethnoraciale par [une] segmentation spatiale de la ville» (WACQUANT, 2012, p. 20). De plus, la ghettoïsation peut être considérée aussi en termes conceptuels. Ainsi, nous retenons la définition de Hamouda (2017):

The conceptual ghetto is a community in which individuals sharing one or more aspect of identity interact with other people of the same community far more than members of other communities. The borders between the members of the conceptual ghetto and others are not physical walls, but they are instead a conceptual understanding of the self and identity (HAMOUDA, 2017, p. 577)

Ainsi, il nous semble important de déterminer quelle est la métaphore qui prime dans la constitution de la littérature des États-Unis, celle du melting pot ou celle du ghetto. Dans ce sens, nous étudierons la configuration du champ littéraire étatsunien, afin de mieux comprendre les enjeux qui caractérisent l'une des plus importantes littératures nationales du monde.

Du point de vue théorique, nous nous basons sur le concept de littérature mineure introduit par Deleuze et Guattari dans Kafka. Pour une littérature mineure (1975). Comme l'affirment les auteurs, " une littérature mineure n'est pas celle d'une langue mineure, plutôt, celle qu'une minorité fait dans une langue majeure» (p. 29). Ainsi, les théoriciens signalent les complexités que renferment les langues pour les immigrants, contexte dans lequel les littératures mineures trouvent leur origine:

Combien de gens aujourd'hui vivent dans une langue qui n'est pas la leur? Ou bien ne connaissent même plus la leur, ou pas encore, et connaissent mal la langue majeure dont ils sont forcés de se servir ? Problème des immigrés et surtout de leurs enfants. Problème des minorités. Problème d'une littérature mineure. (p. 35) 
De toute évidence, cette situation correspond à celle des immigrants qui, depuis de nombreuses décennies, se sont installés, et continuent d'arriver, aux États-Unis. Notre objectif est d'identifier et d'analyser les relations entre les littératures mineures qui ont surgi en sol américain et le champ littéraire national. Spécifiquement, nous analyserons comment les principaux écrivains qui les constituent sont cités et cocités par la critique littéraire académique. En particulier, nous nous attarderons sur la place qu'occupent les écrivaines dans ce contexte.

Dans ce but, du point de vue méthodologique, nous utilisons la criticométrie (FERRER, 2011), approche que Carolina Ferrer a élaborée par analogie à la scientométrie (PRICE, 1963 ; LEYDESDORFF, 1998). L'objectif de la criticométrie est de mesurer et d'analyser l'activité critique en arts, et plus particulièrement en littérature (FERRER, 2017). En ce sens, il s'agit d'une méthode empirique qui se base sur l'exploitation des métadonnées associées aux références bibliographiques contenues dans les bases de données numériques. Spécifiquement, nous avons interrogé la plus importante base littéraire, la Modern Language Association International Bibliography3. Cette base contient plus de 2,8 millions de références et couvre plus de 160 ans de publications effectuées par la communauté académique internationale. En utilisant les termes « littérature française », «littérature anglaise», «littérature canadienne», et cætera dans le champ "littérature nationale», nous avons obtenu les publications pour les 212 nations identifiées par les Nations Unies4. L'échantillon obtenu s'élève à plus de 1,6 million de références et couvre la période de 1844 à 2016.

\section{Cartographie de la littérature des États-Unis}

Selon les dernières recherches de Ferrer (2018) sur la littérature mondiale, la littérature des États-Unis occupe la deuxième place, immédiatement après la littérature du Royaume-Uni. Comme nous pouvons le constater dans le Graphique ${ }^{\circ}{ }^{\circ}$, la littérature des États-Unis cumule plus de

\footnotetext{
${ }^{3}$ Modern Language Association International Bibliography. www.mla.org. Désormais, nous utiliserons I'acronyme MLAIB.

${ }^{4}$ Nous avons identifié plus de 200 nations pour l'année 2017, moment où nous avons interrogé MLAIB. Voir United Nations, http://data.un.org/Default.aspx.
} 
LES ÉCRIVAINES DANS LES LITTÉRATURES MINEURES DES ÉTATS-UNIS: DU MELTING POT Ȧ LA GHETTOÏSATION

280000 publications répertoriées dans la base MLAIB, ce qui correspond à $16,5 \%$ de la littérature mondiale.

Le Graphique $\mathrm{n}^{\circ} 2$ représente la distribution linguistique de la bibliographie critique sur la littérature des États-Unis selon les références contenues dans MLAIB. Les publications en anglais cumulent 90,4\% des données. Viennent ensuite les publications en espagnol, 3,2 \%, en français, $1,8 \%$, en allemand, $1,4 \%$, en japonais, 0,8 \% et en italien, 0,7 \%. Il y a 41 autres langues avec une participation individuelle inférieure à $0,5 \%$ qui se partagent le restant des publications.

Par rapport à la place réservée aux femmes dans le champ littéraire étatsunien, nous pouvons affirmer que celle-ci est très réduite. En effet, si nous considérons les 100 écrivains étatsuniens les plus étudiés selon la base MLAIB, nous observons que les écrivaines occupent une place minoritaire. Comme représenté dans le Graphique $n^{\circ} 3$, les femmes correspondent à seulement $24 \%$ des écrivains les plus étudiés et cumulent $17 \%$ des références de ce sousensemble.

Dans le Tableau $n^{\circ} 1$, nous présentons les 20 écrivains étatsuniens les plus étudiés. Nous avons ajouté le pourcentage de références qu'ils cumulent par rapport au total de la littérature nationale, ainsi que les étiquettes qui leur sont associées. Nous constatons qu'il y a seulement 3 femmes. Il s'agit des écrivaines Emily Dickinson, Toni Morrison et Willa Cather. Aucune ne dépasse $1 \%$ des références.

Le Tableau $n^{\circ} 2$ contient les 20 œuvres littéraires étatsuniennes les plus étudiées selon la base MLAIB. Nous avons identifié les auteurs des œuvres et nous avons inclus les étiquettes qui leur sont associées. Parmi cette vingtaine de titres, 4 sont signées par une femme: Beloved de Toni Morrison, Uncle Tom's Cabin de Harriet Beecher Stowe, The Awakening de Kate Chopin et Their Eyes Were Watching God de Zora Neale Hurston. 


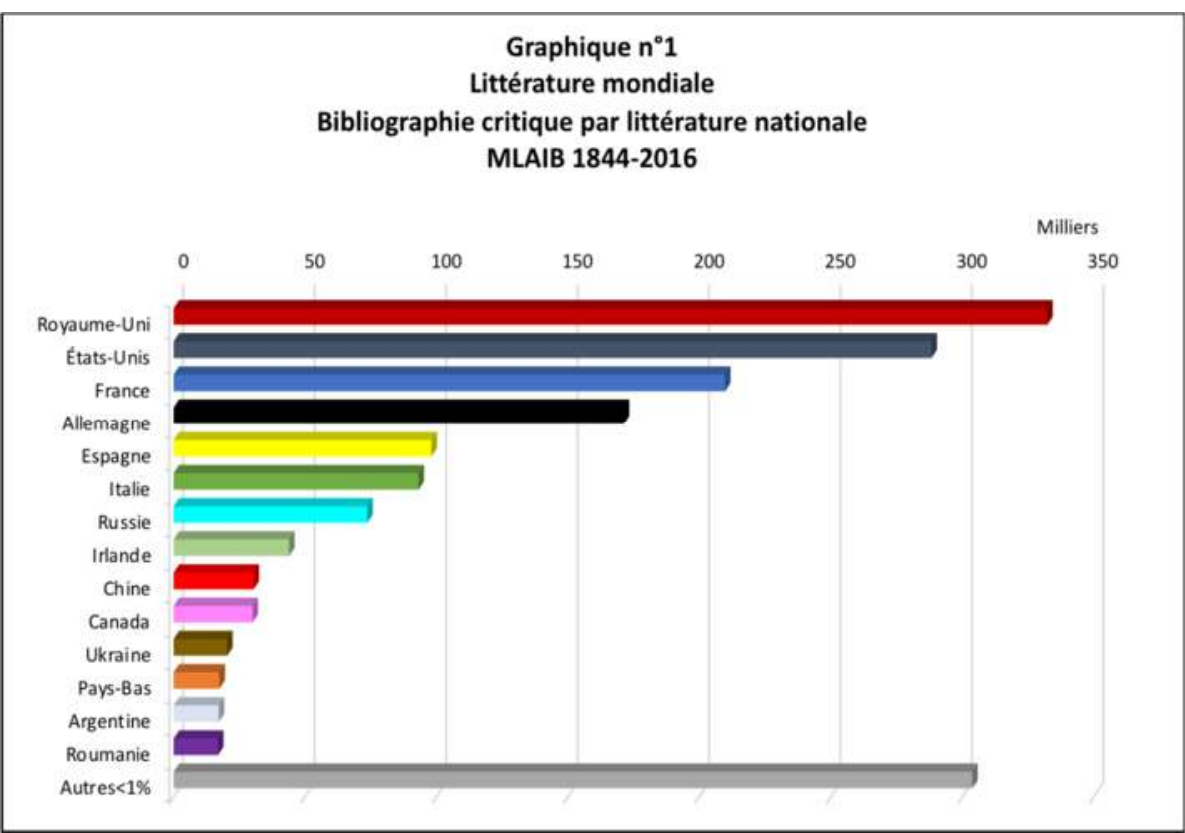

Graphique $n^{\circ} 2$

Littérature des États-Unis

Distribution linguistique de la bibliographie critique MLAIB 1844-2016

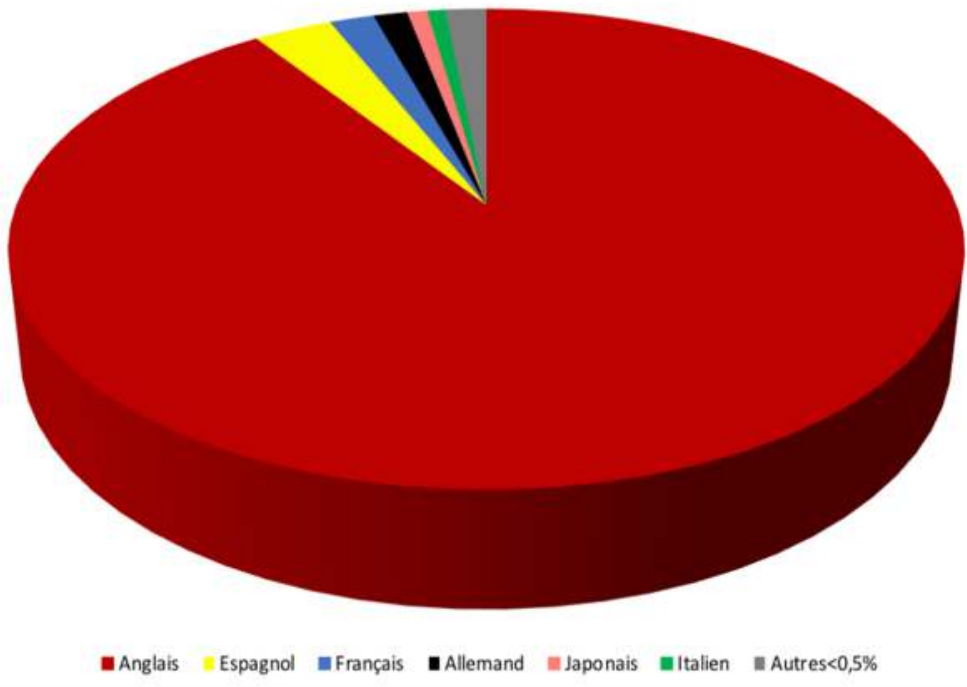


LES ÉCRIVAINES DANS LES LITTÉRATURES MINEURES DES ÉTATS-UNIS: DU MELTING POT Ȧ LA GHETTOÏSATION

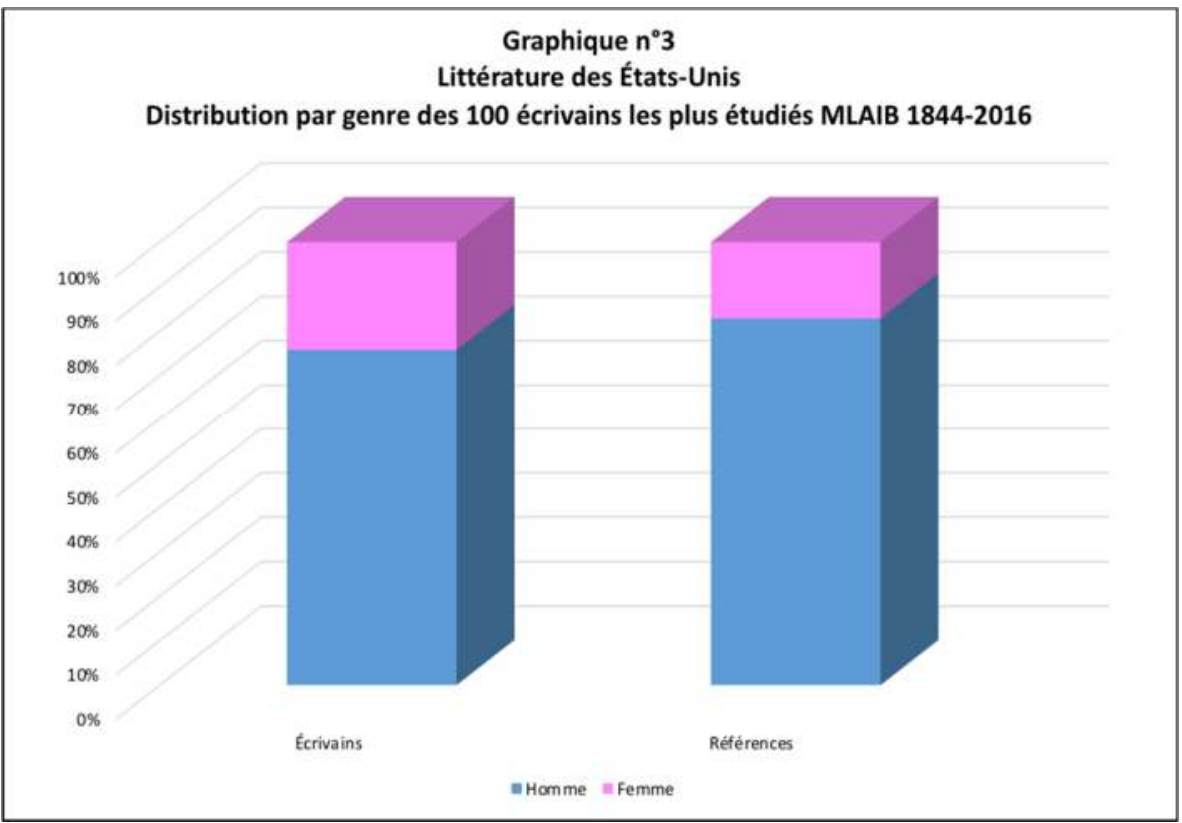

\begin{tabular}{|c|c|c|c|}
\hline \multicolumn{4}{|c|}{ 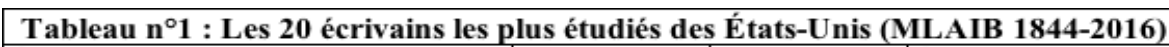 } \\
\hline Écrivain & $\%$ & Genre & Étiquette \\
\hline Faulkner, William (1897-1962) & $2,21 \%$ & Homme & $\mathrm{s} / \mathrm{o}$ \\
\hline James, Henry, Jr. (1843-1916) & $2,15 \%$ & Homme & $\mathrm{s} / \mathrm{o}$ \\
\hline Melville, Herman (1819-1891) & $1,95 \%$ & Homme & $\mathrm{s} / \mathrm{o}$ \\
\hline Hemingway, Ernest (1899-1961) & $1,59 \%$ & Homme & $\mathrm{s} / \mathrm{o}$ \\
\hline Poe, Edgar Allan (1809-1849) & $1,55 \%$ & Homme & $\mathrm{s} / \mathrm{o}$ \\
\hline Clemens, Samuel (1835-1910) & $1,48 \%$ & Homme & $\mathrm{s} / \mathrm{O}$ \\
\hline Hawthorne, Nathaniel (1804-1864) & $1,40 \%$ & Homme & $\mathrm{S} / \mathrm{o}$ \\
\hline \begin{tabular}{|l} 
Whitman, Walt (1819-1892) \\
\end{tabular} & $1,33 \%$ & Homme & $\mathrm{s} / \mathrm{O}$ \\
\hline Pound, Ezra (1885-1972) & $1,16 \%$ & Homme & $\mathrm{s} / \mathrm{o}$ \\
\hline Nabokov, Vladimir (1899-1977) & $1,10 \%$ & Homme & Russian American \\
\hline Emerson, Ralph Waldo (1803-1882) & $0,99 \%$ & Homme & $\mathrm{s} / \mathrm{o}$ \\
\hline Thoreau, Henry David (1817-1862) & $0,98 \%$ & Homme & $\mathrm{s} / \mathrm{o}$ \\
\hline \begin{tabular}{|l|} 
Dickinson, Emily (1830-1886) \\
\end{tabular} & $0,97 \%$ & Femme & $\mathbf{s} / \mathbf{0}$ \\
\hline Fitzgerald, F. Scott (1896-1940) & $0,75 \%$ & Homme & $\mathrm{S} / \mathrm{o}$ \\
\hline Morrison, Toni (1931-) & $0,72 \%$ & Femme & African American \\
\hline Cather, Willa (1873-1947) & $0,71 \%$ & Femme & $\mathbf{s} / \mathbf{0}$ \\
\hline Stevens, Wallace (1879-1955) & $0,68 \%$ & Homme & $\mathrm{S} / \mathrm{o}$ \\
\hline O'Neill, Eugene (1888-1953) & $0,59 \%$ & Homme & $\mathrm{s} / \mathrm{o}$ \\
\hline Frost, Robert (1874-1963) & $0,58 \%$ & Homme & $\mathrm{S} / \mathrm{O}$ \\
\hline Steinbeck, John (1902-1968) & $0,54 \%$ & Homme & $\mathrm{S} / \mathrm{O}$ \\
\hline
\end{tabular}




\begin{tabular}{|c|c|c|c|c|}
\hline \multicolumn{5}{|c|}{ Tableau n² : Les 20 œuvres les plus étudiées des États-Unis (MLAIB 1844-2016) } \\
\hline Euvre & Écrivain & $\%$ & Genre & Étiquette \\
\hline Moby-Dick (1851) & Melville, Herman (1819-1891) & $0,37 \%$ & Homme & $\mathrm{s} / 0$ \\
\hline The Adventures of Huckleberry Finn (1884) & Clemens, Samuel (1835-1910) & $0,29 \%$ & Homme & $\mathrm{s} / 0$ \\
\hline Beloved (1987) & Morrison, Toni (1931-2019) & $0,24 \%$ & Femme & African American \\
\hline The Scarlet Letter (1850) & Hawthorne, Nathaniel (1804-1864) & $0,22 \%$ & Homme & $\mathrm{s} / 0$ \\
\hline The Great Gatsby (1925) & Fitzgerald, F. Scott (1896-1940) & $0,21 \%$ & Homme & $\mathrm{s} / 0$ \\
\hline Lolita (1955) & Nabokov, Vladimir (1899-1977) & $0,19 \%$ & Homme & Russian American \\
\hline Walden (1854) & Thoreau, Henry David (1817-1862) & $0,18 \%$ & Homme & $\mathrm{s} / 0$ \\
\hline Absalom, Absalom! (1936) & Faulkner, William (1897-1962) & $0,18 \%$ & Homme & $\mathrm{s} / 0$ \\
\hline Gravity's Rainbow (1973) & Pynchon, Thomas (1937-) & $0,17 \%$ & Homme & $\mathrm{s} / 0$ \\
\hline The Sound and the Fury (1929) & Faulkner, William (1897-1962) & $0,17 \%$ & Homme & $\mathrm{s} / 0$ \\
\hline Uncle Tom's Cabin (1852) & Stowe, Harriet Beecher (1811-1896) & $0,16 \%$ & Femme & $\mathrm{s} / \mathbf{0}$ \\
\hline Leaves of Grass (1855) & Whitman, Walt (1819-1892) & $0,14 \%$ & Homme & $\mathrm{s} / 0$ \\
\hline Invisible Man (1952) & Ellison, Ralph (1914-1994) & $0,13 \%$ & Homme & African American \\
\hline The Awakening (1899) & Chopin, Kate (1851-1904) & $0,13 \%$ & Femme & French American \\
\hline Light in August (1932) & Faulkner, William (1897-1962) & $0,12 \%$ & Homme & $\mathrm{s} / 0$ \\
\hline The Grapes of Wrath (1939) & Steinbeck, John (1902-1968) & $0,12 \%$ & Homme & $\mathrm{s} / 0$ \\
\hline The Portrait of a Lady (1881) & James, Henry, Jr. (1843-1916) & $0,12 \%$ & Homme & $\mathrm{s} / 0$ \\
\hline The Cantos (1970) & Pound, Ezra (1885-1972) & $0,11 \%$ & Homme & $\mathrm{s} / 0$ \\
\hline Their Eyes Were Watching God (1937) & Hurston, Zora Neale (1891-1960) & $0,11 \%$ & Femme & African American \\
\hline As I Lay Dying (1930) & Faulkner, William (1897-1962) & $0,11 \%$ & Homme & $\mathrm{s} / 0$ \\
\hline
\end{tabular}

\section{Littératures mineures étatsuniennes}

Suite à l'obtention des données sur les publications qui portent sur la littérature des États-Unis, nous avons interrogé à nouveau la base MLAIB, afin d'identifier les étiquettes relatives aux littératures mineures dans lesquelles s'inscrivent certains auteurs. Nous avons obtenu 83 étiquettes associées à 917 écrivains. Parmi celles-ci, nous avons conservé 16 étiquettes selon deux critères de sélection. D'une part, nous avons retenu celles qui contiennent au moins un écrivain avec 10 références ou plus. D'autre part, nous avons sélectionné seulement les étiquettes qui renvoient à une littérature mineure de façon spécifique, éliminant ainsi celles qui se chevauchent les unes avec les autres. À titre d'exemple, l'étiquette Hispanic American contient des écrivains d'origine mexicaine, cubaine, dominicaine et autres. Cette étiquette a été éliminée. Le nombre de références de chaque étiquette retenue est représenté dans le Graphique $n^{\circ} 4$.

La littérature mineure la plus importante est celle qui correspond à l'étiquette African American. Elle représente $48 \%$ des références de l'échantillon. Viennent ensuite les littératures Native American, $14 \%$, et Mexican American, 10 \%. 
LES ÉCRIVAINES DANS LES LITTÉRATURES MINEURES DES ÉTATS-UNIS: DU MELTING POT À LA GHETTOÏSATION

Le Graphique $n^{\circ} 5$ correspond à la chronologie des publications de toutes les étiquettes, par rapport à la bibliographie totale sur la littérature des États-Unis. La série s'étend de 1922 à 2016. Au début, il y a un nombre réduit de publications qui portent sur la littérature identifiée comme Native American. Ensuite, pendant la Deuxième Guerre mondiale, précisément en 1939, l'on commence à voir des références qui correspondent à l'étiquette German American. Cependant, la véritable croissance, toutes étiquettes confondues, commence au début des années 1970, ce qui correspond au début des effervescences sociales, notamment celles reliées au mouvement de revendication de droits civiques5 ainsi qu'aux manifestations contre la guerre du Vietnam et en défense du pacifisme6. Le nombre de publications augmente encore plus fortement à partir de la fin des années 19907. En 2013, la série atteint le maximum avec plus de 1000 documents parus.

Concernant la distribution linguistique, dans le Graphique $n^{\circ} 6$, nous comparons la bibliographie critique sur la littérature étatsunienne totale à celle qui porte sur les littératures mineures. Nous constatons que l'anglais cumule un pourcentage plus élevé de publications que pour l'ensemble des États-Unis: 93,7\% versus 90,4\%. Toutes les autres langues ont une participation inférieure que dans la bibliographie totale. Ceci met en évidence que l'intérêt pour les littératures mineures est principalement d'origine nationale.

\footnotetext{
${ }^{5}$ Voir Hugh Davis GRAHAM. The civil rights era origins and development of national policy: 1960-1972. New York: Oxford University Press, 1990.

${ }^{6}$ Voir Mitchell K. HALL. «The Vietnam Era Antiwar Movement ». OAH Magazine of History, vol. 18, no 5, p. 13-17, 2004.

7 Possiblement, cette croissance est aussi corrélée positivement avec l'augmentation du nombre d'immigrants qui a lieu aux États-Unis à partir des années 1990. Voir Migration Policy Institute. https://www.migrationpolicy.org/data/state-profiles/state/demographics/US\#top, consulté en ligne le 30 août 2019. Par rapport à l'histoire de l'immigration aux États-Unis, voir Roger DANIELS. Coming to America : a history of immigration and ethnicity in American life. New York: Perennial, 2009.
} 

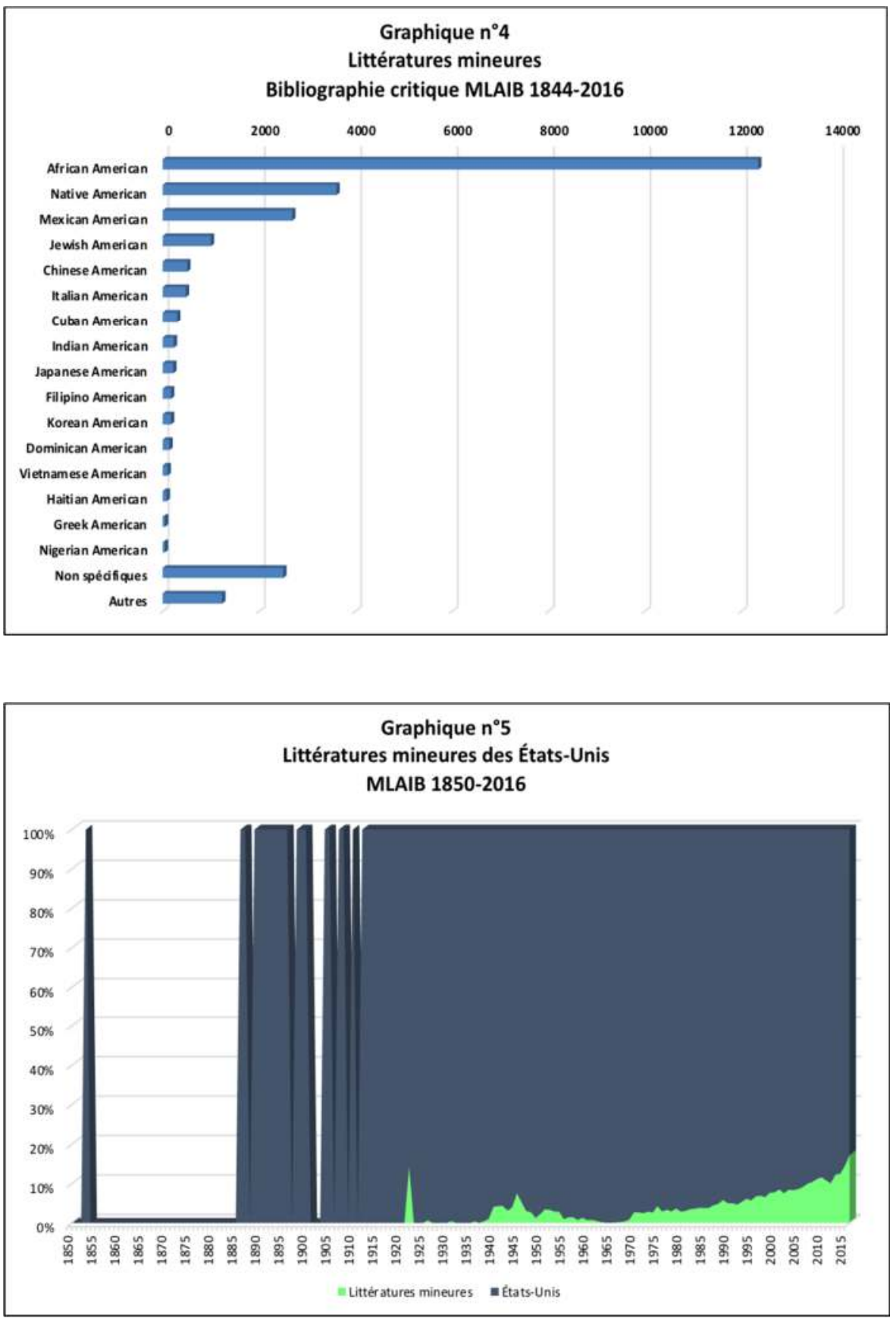


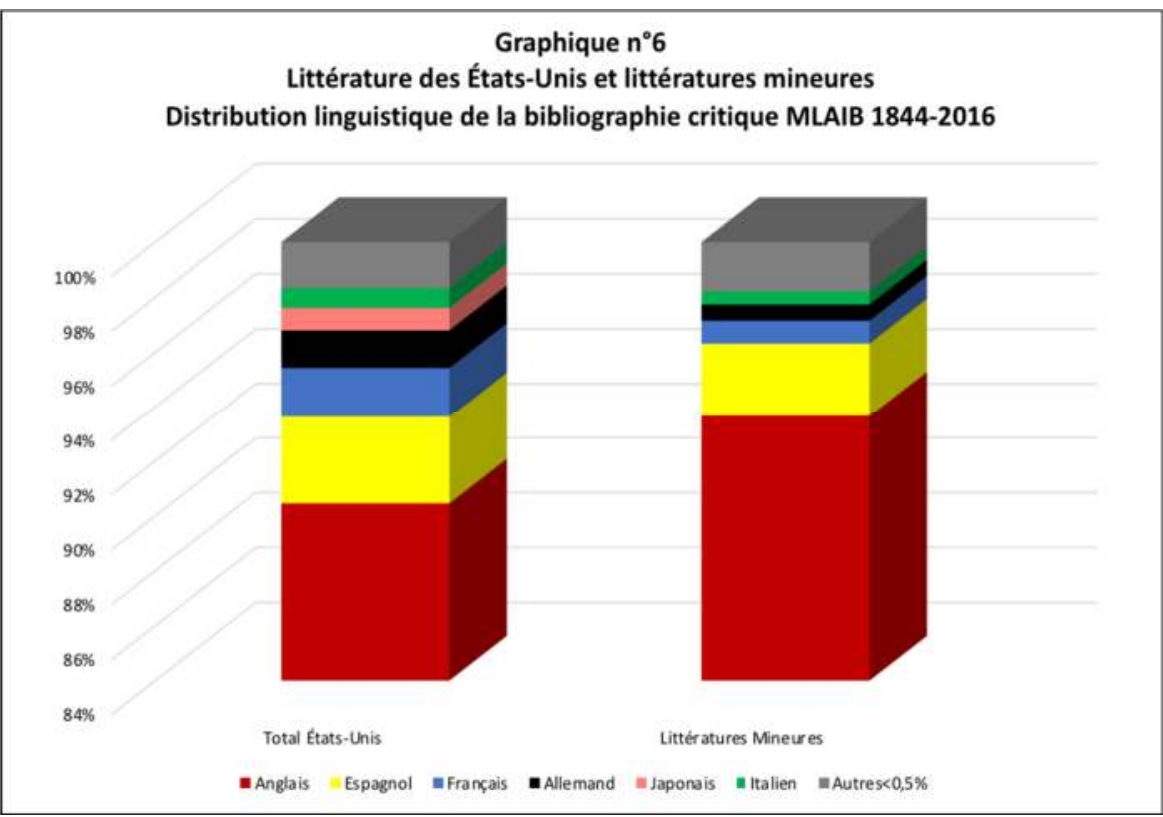

\section{Écrivains sélectionnés}

Afin de mieux comprendre la réception critique des écrivains appartenant aux littératures mineures étatsuniennes, nous avons sélectionné l'écrivain avec le plus grand nombre de références de chacune des étiquettes précises attribuées. Il s'agit d'un ensemble de 16 littératures mineures. Dans le cas de la littérature américaine nigérienne, puisque deux écrivains arrivent ex æquo, nous avons conservé les deux. De plus, nous avons ajouté l'écrivain et l'écrivaine avec le plus grand nombre de références qui ne portent aucune étiquette :William Faulkner et Emily Dickinson.

Le Tableau ${ }^{\circ} 3$ résume les informations de base pour les 19 écrivains sélectionnés. L'échantillon est composé de 12 femmes et de 7 hommes. Immédiatement, nous constatons que dans 10 des 16 littératures mineures analysées, soit $63 \%$ des cas, une écrivaine se trouve au premier rang. Ceci représente une grande différence par rapport à la littérature mondiale et au champ littéraire national où les femmes sont indéniablement marginalisées, comme nous l'avons indiqué ci-dessus. Du point de vue du pays de naissance, malgré leur appartenance à des littératures mineures, 10 des 17 écrivains 
associés à des étiquettes sont nés aux États-Unis (59\%). La langue d'écriture est l'anglais dans $100 \%$ des cas.

Nous observons aussi que les écrivains appartenant aux littératures mineures sont tous nés au 20e siècle et que 12 parmi eux sont encore en vie. En calculant la différence entre la date de naissance de l'écrivain et la date de la première publication critique sur celui-ci, nous constatons une importante variété dans le nombre d'années de décalage. L'écart le plus grand est celui de Papanikolas et le plus petit celui de Danticat.

Le Graphique $n^{\circ} 7$ correspond à la distribution linguistique des publications critiques sur les écrivains sélectionnés. Dans la majorité des cas, l'anglais représente plus de $90 \%$ des publications. Les seules exceptions sont : Fante, dont $24 \%$ de la bibliographie critique est en italien, $5 \%$ en espagnol et $4 \%$ en français; ainsi que trois écrivains avec un pourcentage important de textes en espagnol : Cisneros, $12 \%$, García, $16 \%$, et Díaz, $11 \%$. Nous pouvons ainsi affirmer que même lorsque les écrivains sont associés à une littérature mineure, leur réception se fait essentiellement en anglais.

Afin de mieux cerner les réseaux d'auteurs auxquels les écrivains sélectionnés sont associés, nous élaborons plusieurs indicateurs relatifs aux cocitations. Pour y parvenir, nous avons interrogé à nouveau la base MLAIB, afin d'identifier les écrivains cocités. Nous avons retenu les cocitations qui représentent au moins 0,5\% des références totales sur l'écrivain en question. Le Graphique ${ }^{\circ} 8$ fait état des cocitations du point de vue continental. Il y a peu de comparaisons avec des écrivains à l'extérieur du continent américain. Certains écrivains sont cocités avec des auteurs européens, notamment Fante, Roth et Cole. Plusieurs écrivains appartenant à des littératures mineures d'origine asiatique sont comparés à des écrivains de l'Asie: Bulosan, Lee, Truong, Yamashita et Lahiri. De plus, Roth, Cole, Okorafor et Danticat sont mis en relation avec des auteurs africains. Finalement, Okorafor, Truong, Lahiri et Lee sont cocités avec des écrivains de l'Océanie. La bibliographie sur Cole est la plus diversifiée du point de vue continental des cocitations.

Le Graphique $n^{\circ} 9$ montre les cocitations selon la littérature de provenance des écrivains. À l'exception de Lahiri, Cole, Okorafor et Danticat, les auteurs de l'échantillon sont majoritairement cocités avec des écrivains 
LES ÉCRIVAINES DANS LES LITTÉRATURES MINEURES DES ÉTATS-UNIS: DU MELTING POT À LA GHETTOÏSATION

étatsuniens. En fait, deux écrivains sont comparés exclusivement avec d'autres écrivains étatsuniens: Silko et Papanikolas.

Afin de mieux cerner les relations entre les écrivains qui représentent des littératures mineures étatsuniennes, dans le Graphique ${ }^{\circ} 10$, nous représentons les cocitations par littérature mineure. Faulkner, Dickinson et Cole sont principalement mis en relation avec des écrivains qui n'appartiennent pas à des littératures mineures. La plupart des écrivains sont cocités avec des écrivains qui s'inscrivent dans leur propre littérature mineure. C'est le cas de Silko, Morrison, Roth, Bulosan, Lee, Truong, Lahiri, Cisneros et García. Dans d'autres cas, nous observons que la bibliographie critique met en relation un auteur avec des écrivains associés à plusieurs littératures mineures différentes: Papanikolas, Fante, Kingston, Díaz et Danticat se trouvent dans cette situation.

Le Graphique $\mathrm{n}^{\circ} 11$ correspond aux cocitations par genre. Pour l'ensemble de l'échantillon, nous constatons une répartition homme/femme relativement paritaire. Individuellement, les bibliographies sur l'œuvre de Faulkner, de Dickinson, de Fante, de Roth et de Bulosan cocitent principalement des hommes. Au contraire, celles sur Papanikolas, Kingston, Lahiri, Okorafor, Cisneros, García, Díaz et Danticat font davantage référence à des femmes. Cette situation est très différente de celle observée au niveau national, comme nous l'avons indiqué ci-dessus, puisque les références sur les femmes ne représentent que 17 \% de la bibliographie sur la littérature des ÉtatsUnis.

\begin{tabular}{|l|l|l|l|r|l|r|}
\hline \multicolumn{7}{|c|}{ Tableau $\mathbf{n}^{\circ}$ : Écrivains sélectionnés } \\
\hline \\
\hline Écrivain & $\begin{array}{l}\text { Pays de } \\
\text { naissance }\end{array}$ & $\begin{array}{l}\text { Période } \\
\text { de vie }\end{array}$ & $\begin{array}{l}\text { Langue } \\
\text { d'écriture }\end{array}$ & Réf. & Étiquette & $\begin{array}{r}\text { 1ère réf. } \\
\text { MLAIB }\end{array}$ \\
\hline William Faulkner & États-Unis & $1897-1962$ & Anglais & 6474 & s/o & 1931 \\
\hline Emily Dickinson & États-Unis & $\mathbf{1 8 3 0 - 1 8 8 6}$ & Anglais & $\mathbf{2 8 5 4}$ & s/0 & $\mathbf{1 9 2 6}$ \\
\hline Silko, Leslie Marmon & États-Unis & $\mathbf{1 9 4 8 -}$ & Anglais & $\mathbf{6 6 6}$ & Native American & $\mathbf{1 9 7 8}$ \\
\hline Morrison, Toni & États-Unis & $\mathbf{1 9 3 1 - 2 0 1 9}$ & Anglais & $\mathbf{2 6 4 5}$ & African American & $\mathbf{1 9 7 5}$ \\
\hline Papanikolas, Helen Zeese & États-Unis & $\mathbf{1 9 1 7 - 2 0 0 4}$ & Anglais & $\mathbf{2 1}$ & Greek American & $\mathbf{2 0 0 2}$ \\
\hline Fante, John & États-Unis & $1911-1983$ & Anglais & 75 & Italian American & 1977 \\
\hline Roth, Philip & États-Unis & $1933-2018$ & Anglais & 702 & Jewish American & 1978 \\
\hline Bulosan, Carlos & Philippines & $1914-1956$ & Anglais & 104 & Filipino American & 1984 \\
\hline Kingston, Maxine Hong & États-Unis & $\mathbf{1 9 4 0 -}$ & Anglais & $\mathbf{6 0 9}$ & Chinese American & $\mathbf{1 9 7 8}$ \\
\hline Lee, Chang-rae & Corée du Sud & $1965-$ & Anglais & 147 & Korean American & 1997 \\
\hline Truong, Monique & Vietnam & $\mathbf{1 9 6 8 -}$ & Anglais & $\mathbf{4 3}$ & Vietnamese American & $\mathbf{2 0 0 4}$ \\
\hline Yamashita, Karen Tei & États-Unis & $\mathbf{1 9 5 1 -}$ & Anglais & $\mathbf{1 2 7}$ & Japanese American & $\mathbf{1 9 9 8}$ \\
\hline Lahiri, Jhumpa & Royaume-Uni & $\mathbf{1 9 6 7 -}$ & Anglais & $\mathbf{2 0 8}$ & Indian American & $\mathbf{2 0 0 0}$ \\
\hline Cole, Teju & États-Unis & $1975-$ & Anglais & 48 & Nigerian American & 2013 \\
\hline Okorafor, Nnedi & États-Unis & $\mathbf{1 9 7 4 -}$ & Anglais & $\mathbf{2 7}$ & Nigerian American & $\mathbf{2 0 0 8}$ \\
\hline Cisneros, Sandra & États-Unis & $\mathbf{1 9 5 4 -}$ & Anglais & $\mathbf{3 2 9}$ & Mexican American & $\mathbf{1 9 8 7}$ \\
\hline Garcí, Cristina & Cuba & $\mathbf{1 9 5 8 -}$ & Anglais & $\mathbf{1 8 0}$ & Cuban American & $\mathbf{1 9 9 3}$ \\
\hline Díaz, Junot & Rép. dominicaine & $1968-$ & Anglais & 202 & Dominican American & 2000 \\
\hline Danticat, Edwidge & Haïti & $\mathbf{1 9 6 9 -}$ & Anglais & $\mathbf{3 0 6}$ & Haitian American & $\mathbf{1 9 9 5}$ \\
\hline
\end{tabular}



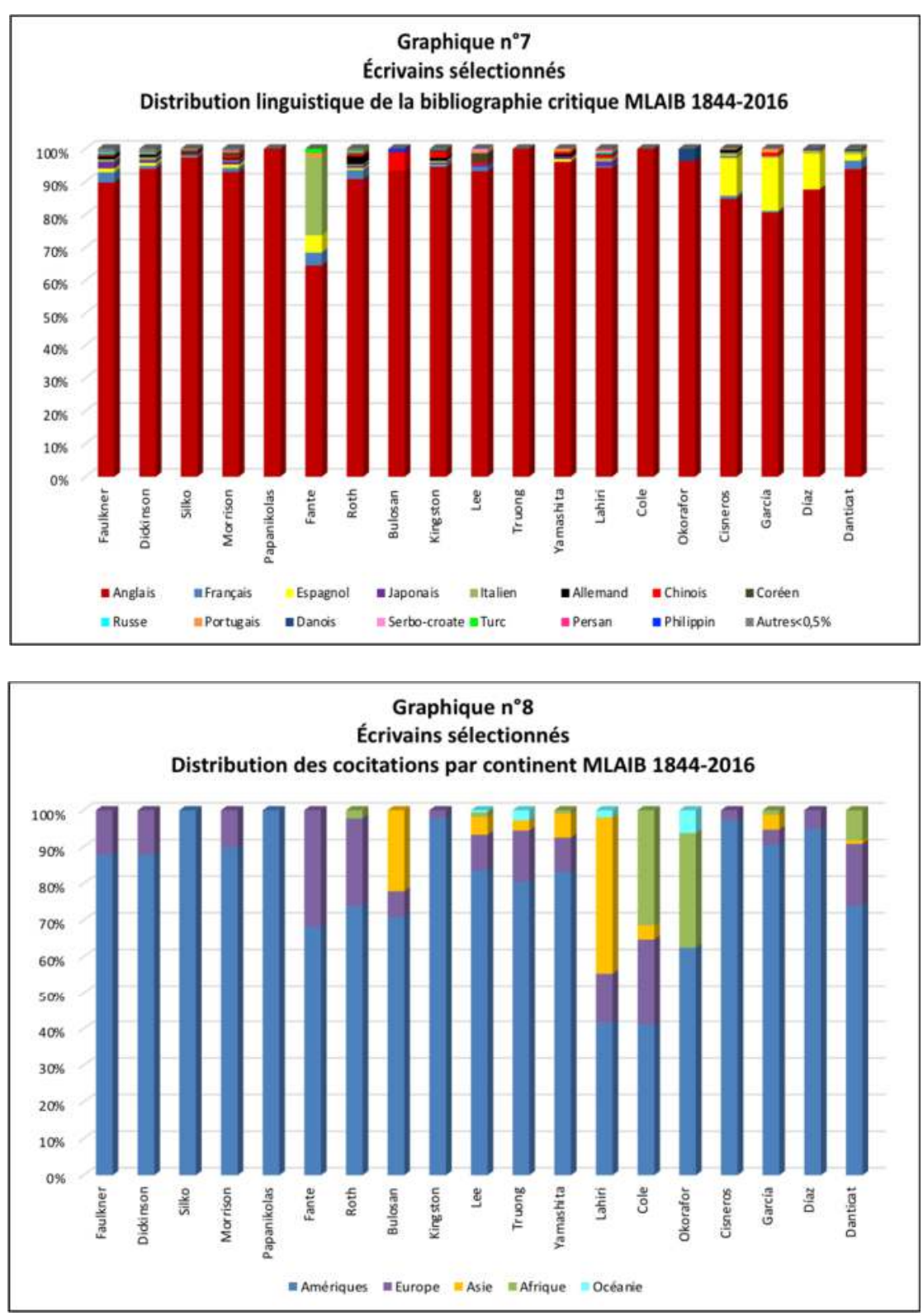
LES ÉCRIVAINES DANS LES LITTÉRATURES MINEURES DES ÉTATS-UNIS:
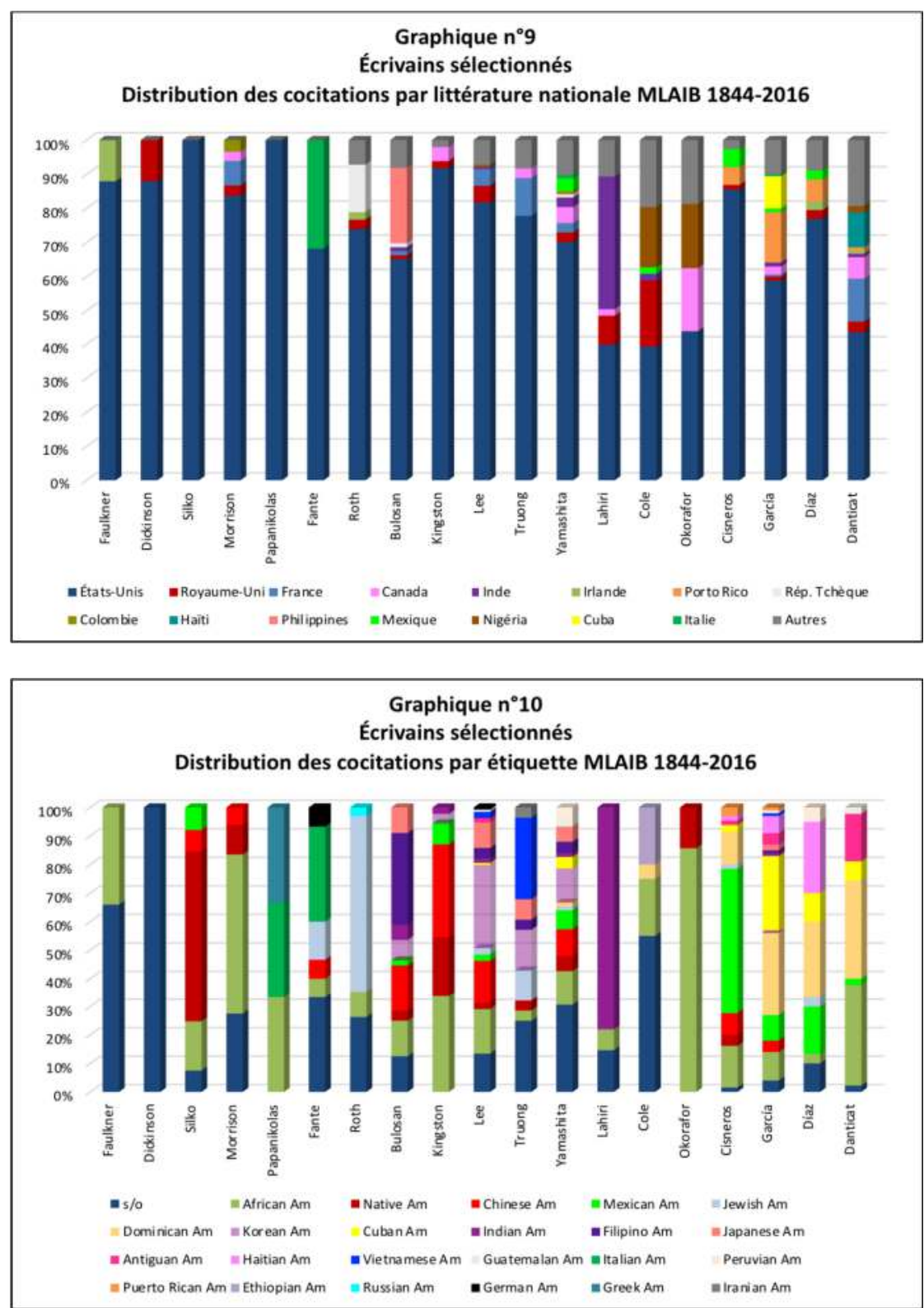

Pontos de Interrogaçăo, v. 9, n. 2, jul.-dez., p. 149-166, 2019. 


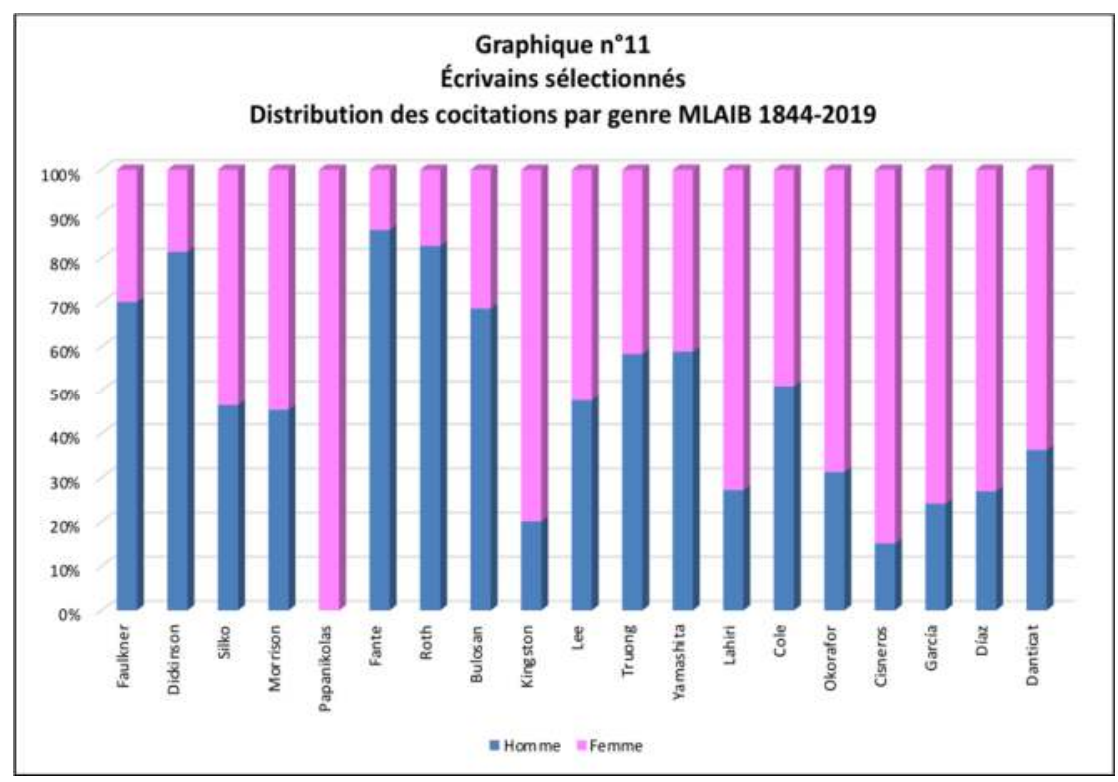

\section{Conclusions}

À travers l'étude des métadonnées des références contenues dans la base MLAIB, nous avons pu identifier les nombreuses littératures mineures qui se sont forgées au sein du champ littéraire des États-Unis. Bien que nous ayons pu confirmer leur présence dès les années 1920, celles-ci deviennent de plus en plus importantes à partir des années 1970.

Du point de vue linguistique, nous observons que la bibliographie critique qui porte sur elles est majoritairement publiée en anglais. Cependant, dans certains cas, les écrivains qui s'inscrivent dans celles-ci sont davantage cocités avec des écrivains étrangers que les auteurs du mainstream étatsunien, comme c'est le cas de Dickinson et Faulkner, qui sont rarement mis en relation avec des écrivains étrangers. Aussi, nous avons constaté que des écrivains de différentes littératures mineures sont mis en relation entre eux à travers les cocitations, ce qui vient renforcer l'idée de ghettoïsation de ces communautés.

Aussi, nous avons détecté une grande présence d'écrivaines parmi les membres les plus représentatifs des différentes littératures mineures. Il semblerait que leur double statut minoritaire, en tant que femmes et en tant que personnes issues de l'immigration, augmente leur visibilité et l'attention que la critique leur accorde. 
LES ÉCRIVAINES DANS LES LITTÉRATURES MINEURES DES ÉTATS-UNIS: DU MELTING POT À LA GHETTOÏSATION

À notre avis, l'analyse criticométrique des données bibliographiques nous permet d'affirmer que, depuis les années 1970, le champ littéraire étatsunien s'éloigne de plus en plus de la métaphore du melting pot et montre des caractéristiques d'une ghettoïsation chaque fois plus profonde.

\section{Références}

ABU-LABAN, Yasmeen et Victoria LAMONT. "Crossing Borders : Interdisciplinarity, Immigration and the Melting Pot in the American Cultural Imaginary». Canadian Review of American Studies/ Revue canadienne d'études américaines, vol. 27, nº 2, p. 23-43, 1997.

DANIELS, Roger. Coming to America: a history of immigration and ethnicity in American life. New York: Perennial, 2009.

DELEUZE, Gilles et Félix GUATTARI. Kafka. Pour une littérature mineure. Paris : Minuit, 1975.

FERRER, Carolina. "El boom hispanoamericano: del texto a la pantalla ». Nuevas aproximaciones al cine hispánico: Migraciones temporales, textuales y étnicas en el bicentenario de las independencias iberoamericanas (1810-2010). Barcelona: Promociones y Publicaciones Universitarias, p. 79-101, 2011.

FERRER, Carolina. "Les études littéraires à l'ère de la mondialisation : traces et trajets au prisme des nouveaux observables numériques". Zizanie, vol. 2, $\mathrm{n}^{\circ}$ 1, p. 76-101, 2018, https://www.zizanie.ca/les-etudes-litteraires-a-lere-de-lamondialisation.html.

FERRER, Carolina. «Étudier la transmission littéraire à l'ère du numérique : des grands écrivains à l'analyse des cocitations ». Trans - Revue de littérature générale et comparée, $\mathrm{n}^{\circ} 22,2017$, http://journals.openedition.org/trans/1719\#article-1719.

GARFIELD, Eugene. "Identifying Core Literature through Citation Analysis and Visualization ", ALA Meeting, Chicago, Committee on Research and Statistics, 2005.

GRAHAM, Hugh Davis. The civil rights era origins and development of national policy: 1960-1972. New York: Oxford University Press, 1990.

HALL, Mitchell K. "The Vietnam Era Antiwar Movement ». OAH Magazine of History, vol. 18, n 5, p. 13-17, 2004. 
HAMOUDA, Yossra. "When Subcultures Turn into Ghettos: The Conceptual Ghetto and Oppression ». Sociology and Anthropology, vol. 5, n 8, p. 577-583, 2017.

LEYDESDORFF, Loet. "Theories of Citation? » Scientometrics, $n^{\circ}$ 43, p. 5-25, 1998.

Migration Policy Institute. https://www.migrationpolicy.org/data/stateprofiles/state/demographics/US\#top, consulté en ligne le 30 août 2019.

Modern Language Association International Bibliography. www.mla.org.

PRICE, Derek de Solla. Little Science, Big Science. New York: Columbia University Press, 1963.

United Nations, http://data.un.org/Default.aspx.

WACQUANT, Loïc. "Les deux visages du ghetto. Construire un concept sociologique ». Actes de la recherche en sciences sociales, vol. 5, n 160, p. 4-21, 2005.

WACQUANT, Loïc. "Repenser le ghetto. Du sens commun au concept sociologique ». Idées économiques et sociales, vol. 1, n 167, p. 14-25, 2012.

Recebido em 20 de março de 2019.

Aceito em 05 de maio de 2019. 